\title{
The Volunteer Satisfaction Index: A Validation Study in the Chinese Cultural Context
}

\author{
Lok Ping Wong • Wing Hong Chui • Yan Yuen Kwok
}

Accepted: 12 September 2010/Published online: 25 September 2010

(C) The Author(s) 2010. This article is published with open access at Springerlink.com

\begin{abstract}
Using a Hong Kong-sourced sample of 261 participants, this study set out to validate the Volunteer Satisfaction Index (VSI) in the Chinese cultural context and to evaluate its psychometric properties. The VSI was originally developed by Galindo-Kuhn and Guzley (2001) to measure the outcomes of volunteer experiences. In this study, exploratory factor analysis (EFA) yielded a different factor structure from that proposed by the scale developer. The three factors found were personal gain, relationship within organization and relationship with peers. Cronbach's alpha values were high for all three subscales. Results from correlation and regression analysis also confirmed the construct and criterion-related validity of the scale. Thus, the reliability and validity of the scale were confirmed. Implications for the assessment of volunteer satisfaction and further directions for cross-cultural studies on related topics are discussed.
\end{abstract}

Keywords Volunteer satisfaction · Volunteer motivation - Scale validation * Hong Kong

\section{Introduction}

Community volunteers are a valuable form of capital for many non-profit organizations engaged in service delivery, as they help reduce the cost of provision and ease the burden on full-time staff (Cemalcilar 2009; Cheung et al. 2006; Clary et al. 1998; Davis et al. 2009; Finkelstein 2008; Finkelstein et al. 2005; Houle et al. 2005; Independent Sector 2001; Penner and Finkelstein 1998; Taylor and Pancer 2007). In volunteer service management, the major challenge agencies face is not recruitment but retention, as reflected by the rapid annual growth rate in the number of volunteers and also the high turnover rates in many countries (Chacon et al. 2007; China Volunteers 2009; Hidalgo and Moreno 2009; Galindo-Kuhn and Guzley 2001; Grimm et al. 2006; Legislative Council Secretariat 2009).

L. P. Wong · W. H. Chui $(\bowtie) \cdot$ Y. Y. Kwok

Department of Social Work and Social Administration, The University of Hong Kong,

Pokfulam Road, Hong Kong

e-mail: ericchui@hku.hk 
The failure to retain volunteers not only has an adverse impact on service provision, but also requires agencies to expend extra effort in recruiting and training new people (Cheung et al. 2006; Galindo-Kuhn and Guzley 2001). Therefore, research has been proposed to investigate the various factors that may help organizations to retain their volunteers.

One of the most frequently-cited such factors is volunteer satisfaction. This has been found to be positively associated with time spent volunteering, longevity of service and intention to continue volunteering (Chacon et al. 2007; Cheung et al. 2006; Clary et al. 1998; Finkelstein 2008; Finkelstein et al. 2005; Galindo-Kuhn and Guzley 2001; Omoto and Snyder 1995; Penner and Finkelstein 1998). Farrel et al. (1998) explained such positive effect on people's volunteering behavior in terms of volunteer motivation: the sense of satisfaction gained in previous volunteering experiences could serve as a motivational force for future voluntary activities. Many other researches indeed found such significant correlation between volunteer satisfaction and volunteer motivation (e.g. Green et al. 1984; Millette and Gagne 2008), suggesting that volunteer satisfaction, volunteer motivation and volunteer experiences are related to one another. Therefore, through continual assessment of the levels of satisfaction perceived by volunteers, and exploration of the source of such positive feelings, agencies will be better able to formulate strategies to motivate further volunteering. This, in turn, could reduce the high dropout rates.

\section{Measuring Volunteer Satisfaction}

To provide researchers and practitioners with a psychometrically sound instrument for assessing volunteer satisfaction, Galindo-Kuhn and Guzley (2001) developed the Volunteer Satisfaction Index (VSI). Volunteers use a seven-point Likert Scale (where $1=$ very dissatisfied; 7 = very satisfied) to indicate their level of satisfaction with different aspects of volunteering.

As opposed to previous studies, which have concentrated on a single dimension of satisfaction using single-item measurements (Chacon et al. 2007; Tschirhart et al. 2001), the VSI is a multi-faceted measure that taps into four dimensions of volunteer job satisfaction. According to this model, satisfaction comes from (a) organizational support, such as performance feedback and clear goals and objectives; (b) participation efficacy, or the use of one's own skills and abilities to make a difference; (c) a sense of empowerment and (d) group integration, or the forming of bonds with other volunteers and paid staff. By going beyond the general concept of volunteer satisfaction, the VSI can help to identify different sources of satisfaction and dissatisfaction in specific domains, so that improvement strategies can be more targeted and therefore more effective. For example, while a one-factor measurement would reflect a general dissatisfaction among volunteers, the VSI might reveal dissatisfaction with a specific domain, such as organizational support. With such detailed information available, agencies can focus on providing more resources and support to enhance the experiences of their volunteers.

Although the VSI is a relatively young instrument, a number of studies have already adopted the multi-dimensional model of volunteer satisfaction as proposed by GalindoKuhn and Guzley (2001). Few of them, however, have evaluated the psychometric properties of the scale (Boezeman and Ellemers 2007; Chacon et al. 2007; Hong et al. 2009; Hume and Hume 2007; Netting et al. 2004; Preston and Brown 2004). Indeed, Galindo-Kuhn and Guzley (2001) themselves only reported the predictive validity and factor analysis of the scale in their original paper, and did not give any reliability statistics, such as the Cronbach's alpha. Thus, more studies are needed to clarify the VSI's psychometric properties. 


\section{Volunteer Sastisfaction in the Chinese Context}

In their scale development study, Galindo-Kuhn and Guzley (2001) proposed a four-factor structure. However, as the scale was originally developed and validated in a western environment, it is uncertain whether this factor structure is also applicable in other cultures.

Previous research on volunteerism in the Chinese context has tended to support the various volunteerism models that originated in the west (Cheung et al. 2006; Wu et al. 2009). Most researchers have focused on evaluating the motives behind volunteering, using the volunteer process model (Omoto and Snyder 1995). There has been little discussion of volunteer satisfaction, even though there is evidence for its influence over voluntary work (Chacon et al. 2007; Cheung et al. 2006; Clary et al. 1998; Finkelstein 2008; Finkelstein et al. 2005; Galindo-Kuhn and Guzley 2001; Omoto and Snyder 1995; Penner and Finkelstein 1998). Even in one study which did include volunteer work satisfaction as one of the variables explaining Chinese volunteers' intention to continue (Cheung et al. 2006), satisfaction was assessed using a single item adopted from the World Values Survey (World Values Study Group 1990). Thus the study could only address overall satisfaction, rather than identifying specific sources of satisfaction and dissatisfaction. Therefore, to facilitate local research and best practice in volunteer retention, more studies are needed to validate the VSI and the multi-dimensional model of volunteer satisfaction (Galindo-Kuhn and Guzley 2001) in the context of Chinese culture.

\section{Aims of the Study}

The purpose of this study is two-fold: firstly, to investigate whether the factor structure proposed by Galindo-Kuhn and Guzley (2001) is applicable in the Chinese cultural context; and secondly, to evaluate the psychometric properties of the Chinese version of the VSI (VSI-C).

Galindo-Kuhn and Guzley (2001) proposed four factors underlying volunteer satisfaction; organizational support, participation efficacy, empowerment and group integration. Since their study was conducted in the western cultural context, it is uncertain whether their results can be generalized to other settings. By performing a factor analysis using data collected from a Hong Kong Chinese sample, the current study aims to evaluate whether the factor structure arrived at in this way similar to or different from that previously obtained from the western study. This has the potential not only to improve our understandings of volunteerism in the Chinese culture, but also to facilitate further cross-cultural research of related issues.

In addition to investigating the factor structure of the VSI-C, this study also aims to evaluate its psychometric properties. As mentioned previously, although Galindo-Kuhn and Guzley (2001) provided evidence for the scale's predictive validity and factor structure, they did not evaluate its reliability. Also, no other studies since then have examined its psychometric properties. Accordingly, the second aim of this study is to fill the gap by assessing the internal reliability, construct validity and criterion-related validity of the VSI-C.

In evaluating the scale's validity, a few hypotheses have been generated. First, to assess the construct validity of VSI-C (i.e. the extent to which it measures the theoretical concept of volunteer satisfaction), a bivariate correlation analysis between VSI-C and an instrument tapping into volunteer motivation will be performed. It is hypothesized that there will be significant and positive correlation between the two concepts, as they are both important 
theoretical constructs within volunteerism. In fact, previous researches have already pointed to a positive correlation between the two variables (see, for example, Chacon et al. 2007; Chapman and Morley 1999).

Previous research has demonstrated that many of the volunteering behaviors could be predicted by volunteer satisfaction, such as time to be spent in volunteering (e.g. Finkelstein 2008), we believe that the VSI-C score could also predict similar volunteering behaviors or decisions. Specifically, it is hypothesized that the more satisfied a volunteer is, the more target groups and organizations s/he would serve, and the more time s/he would spend volunteering.

\section{Method}

\subsection{Participants}

A total of 261 university students with previous volunteering experience (185 females and 76 males), ranging in age from 18 to 66 with a mean of $25.6(\mathrm{SD}=8.90)$, were recruited from four tertiary institutions in Hong Kong. Three hundred and forty-eight copies of the questionnaires were distributed at the beginning of four professors' class, and these students were given 20 minutes to complete the questionnaire. As participation was voluntary, some did not complete the entire questionnaire and some did not return the questionnaire. The final response rate was approximately $75 \%$.

After their consent had been obtained, questionnaires were delivered to the participants either in hard copy or through an Internet site. To ensure that the form of questionnaire distribution would not affect the results, an independent $t$-test was performed to compare the hard copy $(n=186)$ with the Internet $(n=76)$ groups on all major measurements. No significant difference in scores was found, so no statistical adjustment for the form of distribution was needed in the subsequent analysis.

\subsection{Measures}

As well as the VSI-C as described above, a volunteer motivation scale and some other questions measuring volunteering-related experiences and demographic information were also included for validation purposes. Since all our respondents were native Chinese speakers, all scales used were translated into Chinese by a research assistant who was blind to the hypotheses and design of this study. These scales were then back translated into English by another research assistant so as to enhance methodological rigor. Besides the back-translation procedure, a social science professor and a trained interpreter who are fluent in both Chinese and English had helped to proof-read the translated version in order to ensure VSI-C's equivalence in meaning with the original VSI. A pilot study was also conducted to test ordinary participants' understanding towards the translated VSI-C. This consists of 26 items, with responses given using a 7-point Likert Scale (where $1=$ strongly dissatisfied and $7=$ strongly satisfied). In a validation study conducted by the original scale developers, four dimensions of volunteer job satisfaction were derived, namely organizational support, participation efficacy, empowerment and group integration (see Table 1 for a detailed explanation of each subscale). A previous study (Galindo-Kuhn and Guzley 2001) showed satisfactory alpha coefficients for all subscales, ranging from 0.75 to 0.91 . 
Table 1 Detailed explanation of each subscale of the volunteer satisfaction index (VSI) (Galindo-Kuhn and Guzley 2001) and the Volunteer Function Inventory (VFI) (Clary and Snyder 1999)

\begin{tabular}{ll}
\hline Subscales & Explanation \\
\hline VSI-Organizational support & $\begin{array}{c}\text { Derive satisfaction through educational and emotional } \\
\text { resources provided by the organization } \\
\text { Gain satisfaction through having the ability to bring about } \\
\text { good changes to clients }\end{array}$ \\
VSI-Empowerment & Feeling empowered during one's service \\
VSI-Group integration & $\begin{array}{l}\text { Gain satisfaction through forming social relationships with } \\
\text { other volunteers and paid staff }\end{array}$ \\
VFI-Values & To express important values, e.g. altruism \\
VFI-Understanding & To gain new experiences \\
TFI-Enhancement & To exercise unpracticed skills and abilities \\
VFI-Career & To gain personal growth and development \\
VFI-Social & To obtain career-related experiences \\
VFI-Protective & To strengthen one's social relationships \\
& To reduce negative feelings (e.g. guilt) \\
& To address personal problems
\end{tabular}

Volunteer Functions Inventory (VFI) (Clary and Snyder 1999). This instrument contains 30 items and 6 subscales measuring the reasons for volunteering. It would be used to validate the VSI-C. Each subscale includes five items that tap into one of the volunteering motives, such as values, understanding, social, career, protective and enhancement (see Table 1 for a detailed explanation of each subscale). Each item was measured using a 7-point Likert scale (where $1=$ not at all important/accurate and $7=$ extremely important/accurate). A sample item is "I am concerned about those less fortunate than myself" (social motive). A validation study of VFI using a local Chinese student sample has conducted (Wu et al. 2009) and demonstrated a good internal consistency, with reliability coefficients ranging from 0.70 to 0.91 .

Voluntary Experiences: In addition to the VFI, several questions on voluntary experiences were also asked for validation purpose. Participants were asked to indicate the number of target groups and, number of organizations they had been involved with, and the average number of hours per month they had given, over the past 12 months.

Demographic Variables: Relevant demographic characteristics including gender, age, marital status and religion were collected.

\section{Results}

\subsection{Exploratory Factor Analysis}

To evaluate whether the original factor structure proposed by the scale developer (GalindoKuhn and Guzley 2001) could be transferred to the Chinese context, an EFA was performed.

The number of valid cases for the EFA was 236. The Kaiser-Meyer-Olkin (KMO) test and Bartlett's test of sphericity were carried out to assess the appropriateness of using factor analysis on the dataset. The KMO value was 0.92 , which was greater than the required value of 0.5 . The Bartlett's test of sphericity was found to be significant with a 
$p$ value $<0.001\left(x^{2}=4420.2, d f=325\right)$. These results indicated that it was appropriate to conduct an EFA.

All 26 items of the full scale of VSI-C were subjected to a principal component factor analysis with Varimax rotation. Under the criterion of eigenvalues greater than 1 and supported by the scree test (Cattell 1966), four dimensions were found, each with an eigenvalue exceeding 1 . Taken together, these four factors accounted for $66.24 \%$ of the total variance. Factor loadings after Varimax rotation of each item are presented in Table 2. Since the fourth factor consisted of only two items (items 25 and 26), and could not be easily interpreted, it was dropped from the scale entirely.

There were certain items which had a loading of 0.40 or above on more than one factor. To tackle this issue of double loading, the Thurstone's Simple Structure rule

Table 2 Factor loadings after varimax rotation of each item

Items

Factor loadings

$1 \quad 2 \quad 3 \quad 4$

1. The amount of information I receive about what the organization is doing

2. The flow of communication coming to me from paid staff and board members .763

3. The support I receive from people in the organization $\quad .744$

4. The way in which the agency provides me with performance feedback $\quad .724$

5. How often the organization acknowledges the work I do 723

6. My relationship with paid staff $\quad .706$

$\begin{array}{llll}\text { 7. The degree to which the organization communicates its goals and objectives } & .673 \quad .521\end{array}$ to volunteers

8. The support network that is in place for me when I have volunteer-related $\quad 667$ problems

9. The amount of permission I need to get to do the things I need to do on this job

10. The degree of cohesiveness I experience within the organization

11. The difference my volunteer work is making

12. The chance I have to utilize my knowledge and skills in my volunteer work

13. My ability to do this job as well as anyone else.

14. How worthwhile my contribution is

15. The amount of effort I put in as equaling the amount of change I influence

16. The opportunities I have to learn new things

17. The fit of the volunteer work to my skills

18. The progress that I have seen in the clientele served by my organization

19. The access I have to information concerning the organization

20. The freedom I have in deciding how to carry out my volunteer assignment

21. The amount of interaction I have with other volunteers in the organization

22. The friendships I have made while volunteering here

23. The amount of time spent with other volunteers in the organization

26. The realism of the picture I was given of what my volunteer experience would be 
(Thurstone 1953) was applied, which states that if the percentage difference between the loadings is greater than 0.05 , the item can be considered to belong primarily to the factor with the higher loading.

After these statistical adjustments had been completed, we proposed a three-factor structure for the VSI-C. Factor 1 consisted of 10 items, which measured the satisfaction derived from interacting with members of the volunteering organization. This was labeled relationship within organization. Factor 2 also consisted of 10 items, these being related to the personal gain of volunteers and involving, autonomy, personal growth and the sense of satisfaction gained through making a contribution. This factor was labeled as personal gain. The final factor consisted of four items, which measured the satisfaction occasioned by forming relationships with volunteering peers. It was labeled relationship with peers. The factor loadings of this final structure are presented in Table 3.

\subsection{Reliability Analyses}

Internal reliability coefficients for the personal gain, relationship within organization and relationship with peers subscales, as computed by the Cronbach's alphas, were $0.91,0.6$

Table 3 Factor loadings of the finalized structure

Items $\quad \begin{array}{ll}\frac{\text { Factor loadings }}{} \\ \text { I }\end{array}$

1. The amount of information I receive about what the organization is doing

2. The flow of communication coming to me from paid staff and board members

3. The support I receive from people in the organization

4. The way in which the agency provides me with performance feedback

5. How often the organization acknowledges the work I do

6. My relationship with paid staff

7. The degree to which the organization communicates its goals and objectives to volunteers

8. The support network that is in place for me when I have volunteer-related problems .667

9. The amount of permission I need to get to do the things I need to do on this job $\quad .654$

10. The degree of cohesiveness I experience within the organization

11. The difference my volunteer work is making

12. The chance I have to utilize my knowledge and skills in my volunteer work

13. My ability to do this job as well as anyone else

14. How worthwhile my contribution is

15. The amount of effort I put in as equaling the amount of change I influence

16. The opportunities I have to learn new things

17. The fit of the volunteer work to my skills

18. The progress that I have seen in the clientele served by my organization

19. The access I have to information concerning the organization

20. The freedom I have in deciding how to carry out my volunteer assignment

21. The amount of interaction I have with other volunteers in the organization

22. The friendships I have made while volunteering here

23. The amount of time spent with other volunteers in the organization 
and 0.93 respectively. On the subscale level, the three dimensions were significantly related to each other and to the total VSI-C score. On the item level, the within-scale inter-item correlations were all positive and significant (see Table 4). Additionally, all within-scale corrected item-total correlations were positive, with means ranging from 0.81 to 0.83 .

\subsection{Construct Validity}

Correlations among the total and subscale scores of the VSI-C, and the total score of VFI, are shown in Table 4. As expected, all motivational measurements positively correlated with all satisfaction measurements. According to the criteria as suggested by Cohen (1988), the correlation coefficients are of a small effect size (i.e. around 0.2). In other words, the reasons for volunteering and the source of volunteer satisfaction have a small to medium and positive association with each other.

\subsection{Criterion-Related Validity}

To examine the appropriateness of using the VSI-C to statistically predict volunteering behavior, three sets of linear regressions were performed. The VSI-C total was entered into each set as an independent variable, while the number of target groups, number of organizations and average hours per month given by the volunteers within the past 12 months were all used as outcome variables. The results suggested that the VSI-C total score is a statistically significant predictor of all three criteria (see Table 5). $R^{2}$ values for the three equations were all 0.05, which, according to Cohen's criteria (1988), indicated a small to medium effect size.

\section{Discussion}

The purpose of our study was to provide a reliable and valid measure, suitable for use with the Hong Kong Chinese population, of factors influencing volunteers' satisfaction. Our results have not only demonstrated that the VSI-C is a psychometrically sound measure of volunteer satisfaction, but have also revealed a factor structure unique to the Chinese population.

\subsection{Unique Three-Factor Structure}

Our initial EFA yielded a three-factor structure quite different from that proposed originally by the scale developer. We believe that the difference between these two models is not merely a matter of the number of factors proposed, but of their content and the underpinning cultural implications.

The four factors uncovered by Galindo-Kuhn and Guzley (2001) focused more on the personal benefits gained from volunteering, such as feelings of empowerment and the organizational support obtained. Of these four factors, only one (group interaction) focused on relationship building. In contrast, two of the three factors proposed by this study pertain to interpersonal relationships (relationship with organization and with peers). Only one factor focuses on personal gain, such as enhanced autonomy. 


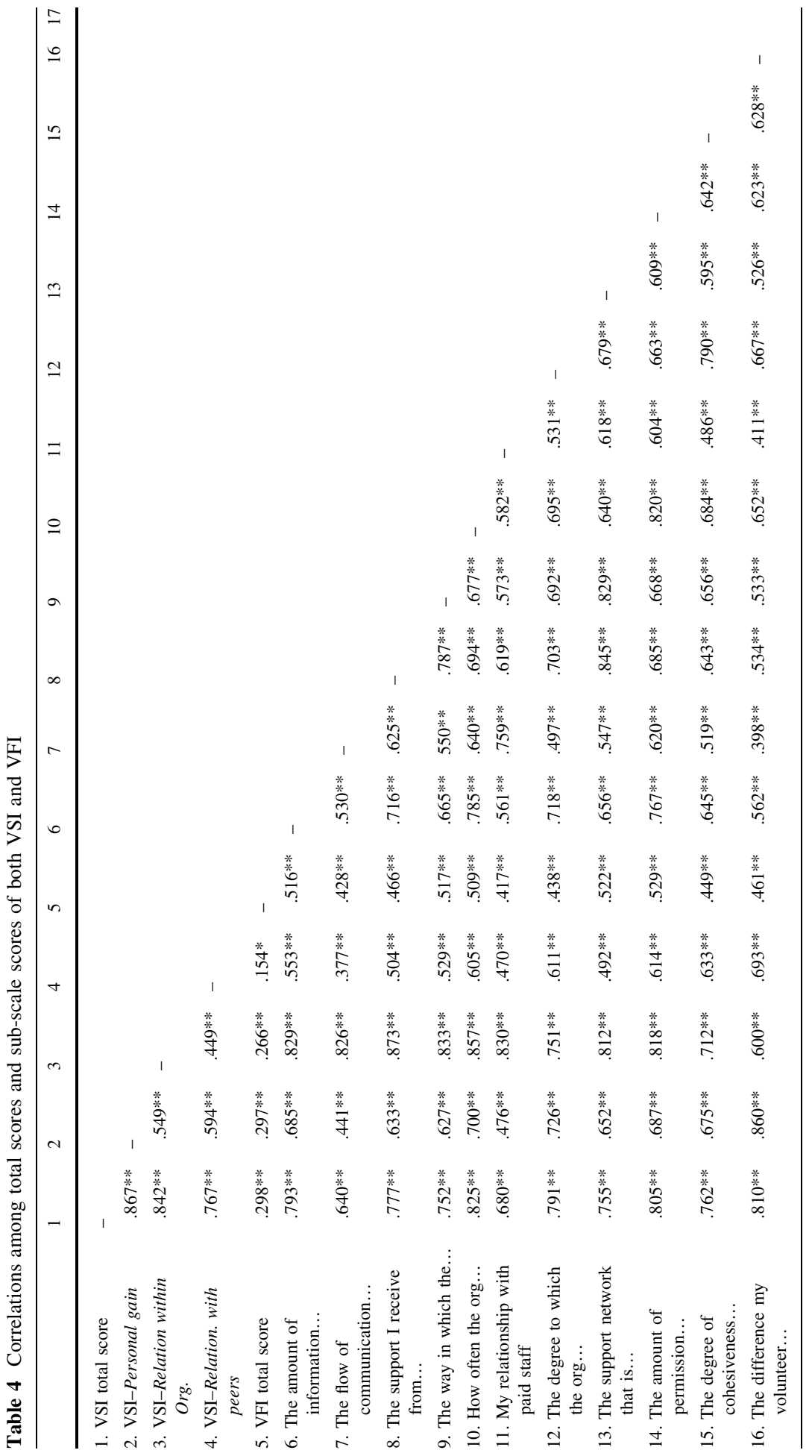




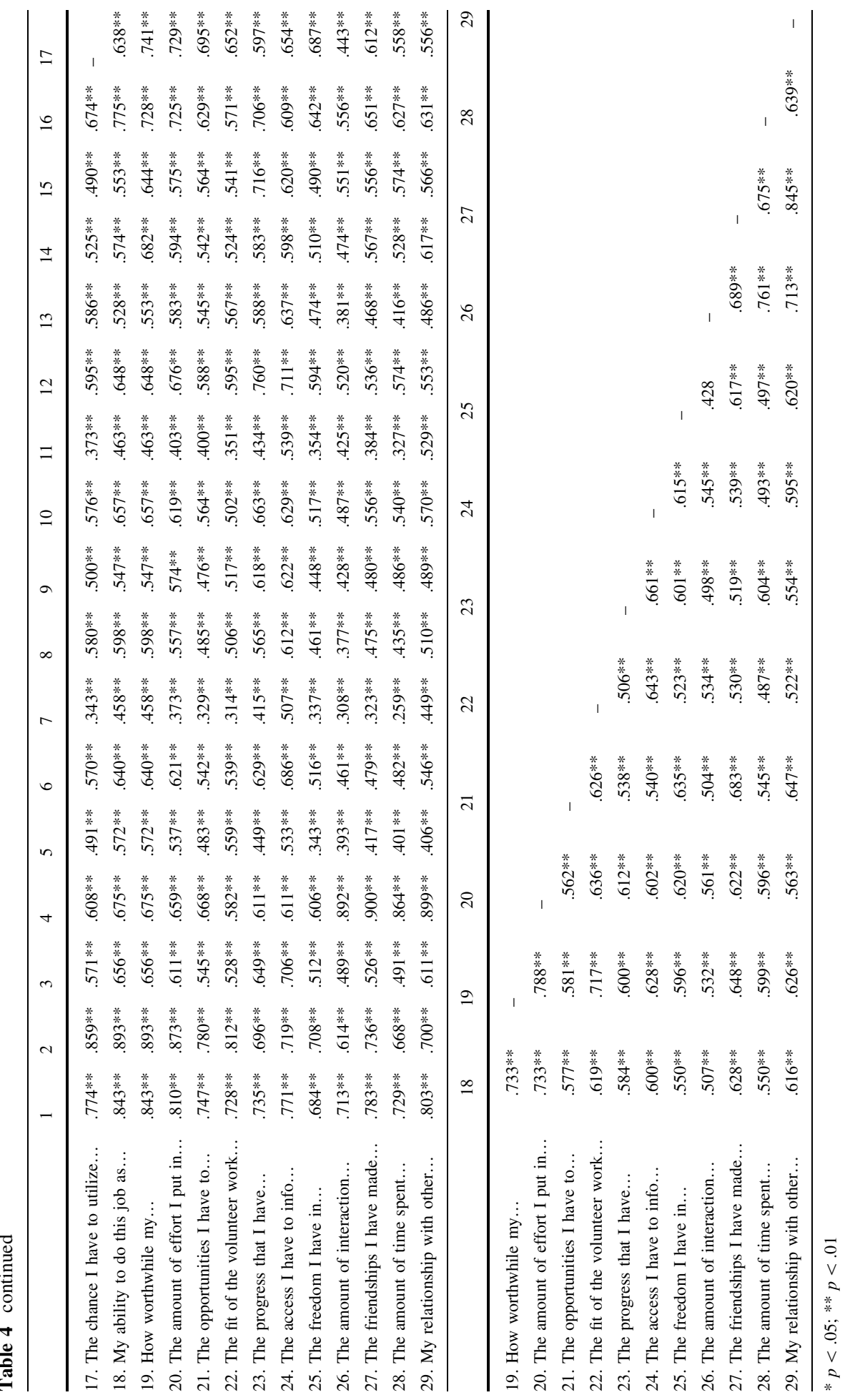


Table 5 Results of linear regression with VSI-C total as the predictor

\begin{tabular}{lllllll}
\hline Criterion & $b$ & SE of $b$ & Beta & Tolerance & $t$ & $p$ \\
\hline No. of target groups & .251 & .053 & .226 & 1.00 & 4.74 & .000 \\
No. of org. served & .229 & .048 & .229 & 1.00 & 4.80 & .000 \\
Hrs. per month served & .390 & .087 & .214 & 1.00 & 4.49 & .000 \\
\hline
\end{tabular}

Additionally, while efficacy, the ability to bring about positive changes for clients, stood alone as one factor in the original study, it is no longer an independent factor in our current research but instead has merged with empowerment to form the personal gain factor.

The differences in the cultural context of the two studies might explain this divergence in factor structure. While Galindo-Kuhn and Guzley (2001) first conducted their study in the west, where the majority of their sample consisted of Caucasians (83\%), the present study recruited participants from the Hong Kong Chinese community. Thus, the unique factor structure we have might be explained in terms of the Chinese cultural characteristics. According to Confucianism, an individual is fundamentally a relational being (Farh et al. 2008). In such a relationship-oriented world, social relations are so important that they alone can serve as a source of satisfaction. This might explain why our Chinese participants put such great emphasis on interpersonal relationships and understated personal gain as a source of volunteer satisfaction.

Furthermore, it has been reported that Chinese culture tends to categorize people and treat them accordingly (Farh et al. 2008). Such social categorization might explain why the idea relationships, as a source of volunteer satisfaction, is further broken down into relationships with the organization and with peers, instead of both types of interaction being combined into the broader category of group integration as in the original western sample.

\subsection{Good Psychometric Properties}

Our results have also showed that the VSI-C has good internal consistency. Firstly, the alpha values of all subscales exceeded 0.60. According to Moss et al. (1998), an alpha score of 0.6 is generally acceptable, even though such cutoff might not be as stringent as the 0.7 threshold (Nunnally 1978; Sturmey et al. 2005). There were indeed quite a number of studies which adopted the 0.6 criterion (e.g. Haher et al. 1999; Morgan et al. 2004; Reader et al. 2007). Hence, the internal reliability of the VSI-C is justifiable.

In addition to the acceptable level of the Cronbach's alpha for each subscale, the strong subscale-to-total correlations indicated that the scale as a whole is a reliable measurement. Secondly, the high within-subscale item-total and inter-item correlations not only further confirmed the finding of internal reliability, but also provided stronger support for the three-factor structure proposed in this study.

In the evaluation of the VSI-C's construct validity, all subscales in the VFI showed a positive correlation with all the VSI-C subscales. This suggests that the things which motivate people to volunteer are positively associated with what satisfies them afterward. This pattern of convergence is consistent with the theoretical conceptualization of volunteering as discussed in previous studies (Chacon et al. 2007; Chapman and Morley 1999) and might be further examined by using VSI-C in further studies in the Chinese population.

As well as demonstrating construct validity through its convergence with volunteer motivation, the VSI-C also showed criterion-related validity, being able to statistically predict the number of target groups and organizations the person was involved with, and 
the average amount of hours per month they have given, over the past 12 months. Specifically, the more satisfied the volunteer is, the more target groups and organizations he or she will serve, and the more time he or she will give. This is consistent with the pattern of findings obtained in the original VSI research (Galindo-Kuhn and Guzley 2001), where volunteer satisfaction predicted future volunteering decisions. In fact, the result is also consistent with findings from other studies that satisfaction predicts the time people will spend volunteering (Finkelstein 2008). However, it should be noted that with a crosssectional design, such statistically proven predictive powers of volunteer satisfaction do not allow us to draw up a casual relationship among the variables. To establish a casual relationship, future longitudinal design should be used.

\subsection{Future Directions and Conclusion}

The current study is the only one to have evaluated the constructs underlying the satisfaction of Hong Kong Chinese volunteers. It demonstrates that due to cultural variation, they derived satisfaction from quite different sources than their western counterparts. Specifically, while relationship building is a major component of volunteer satisfaction in Chinese culture, personal benefits tend to be what satisfy western volunteers. Such a cultural difference should shed light on directions for further research on the property of the scale. For example, qualitative research could be conducted to see whether there are further unique factors or concepts underlying volunteer satisfaction in the Chinese culture that have not been identified using the current 24 items. Also, further studies could be conducted in other Chinese populations such as those in Taiwan and the Mainland to see if the same findings apply.

As the VSI-C has already been validated in Hong Kong, researches concerning volunteer satisfaction and its correlates in the local context are now possible. One relevant research direction is to test whether and how some of the Chinese cultural characteristics such as the doctrine of the mean (zhong yōng) may influence Chinese volunteers' satisfaction. The doctrine of the mean is a Confucian concept that gives guidelines to how men could perfect oneself (Fu and Cai 2009). There are indeed many principles underlying this broad concept that might influence Chinese volunteers' satisfaction. For example, the doctrine advocates the expression of emotions in an appropriate manner. To put this into the volunteering context, it would mean that even when one feels s/he has obtained great personal gain from volunteering, one should not express great satisfaction when asked, for fear that this would be a sign of "arrogance" that is not appropriate. Similarly, they would also avoid showing great dissatisfaction, for fear that this would cause the organization or colleagues to "lose face". With the VSI-C readily available, we could examine how response style to the VSI-C relates to one's level of conformity to the doctrine of the mean's teachings. It is hypothesized that the more one agrees and internalizes such teachings, the less likely that one would express an extreme level of volunteering satisfaction or dissatisfaction.

In conclusion, the present study has gone beyond translating and validating the VSI-C. Now that its satisfactory psychometric properties have been demonstrated, the VSI-C can now be used for research, assessment and volunteer retention/training purposes in the Hong Kong Chinese context. Moreover, through the discovery of the new factor structure, this study sheds light on directions for further cross-cultural research on volunteer satisfaction and motivation.

Acknowledgments We are grateful to Kit Fok and Timothy Yan for their support with data collection. 
Open Access This article is distributed under the terms of the Creative Commons Attribution Noncommercial License which permits any noncommercial use, distribution, and reproduction in any medium, provided the original author(s) and source are credited.

\section{References}

Boezeman, E. J., \& Ellemers, N. (2007). Pride and respect in volunteers' organizational commitment. European Journal of Social Psychology, 38, 159-172.

Cattell, R. B. (1966). The scree test for the number of factors. Multivariate Behavioral Research, 1, 245-276.

Cemalcilar, Z. (2009). Understanding individual characteristics of adolescents who volunteer. Personality and Individual Differences, 46, 432-436.

Chacon, F., Vecina, M. L., \& Davila, M. C. (2007). The three-stage model of volunteers' duration of service. Social Behavior and Personality, 35, 627-640.

Chapman, J. G., \& Morley, R. (1999). Collegiate service learning: Motives underlying volunteerism and satisfaction with volunteer service. Journal of Prevention and Intervention in the Community, 18, 19-33.

Cheung, F. Y., Tang, C. S., \& Yan, E. C. (2006). Factors influencing intention of continue volunteering: A study of older Chinese in Hong Kong. Journal of Social Service Research, 32, 193-208.

China Volunteers (2009). Youth volunteers report 2009 (In Chinese). Retrieved from http://www.zgzyz.org. $\mathrm{cn} /$ volunteer/content.jsp?id=67716\&cid=20056.

Clary, E. G., \& Snyder, M. (1999). The motivations to volunteer: Theoretical and practical considerations. Current Directions in Psychological Sciences, 8, 156-159.

Clary, E. G., Snyder, M., Ridge, R. D., Copeland, J., Stukas, A. A., Haugen, J., et al. (1998). Understanding and assessing the motivations of volunteers: A functional approach. Personality Processes and Individual Differences, 74, 1516-1530.

Cohen, J. (1988). Statistical power analysis for the behavioral sciences (2nd ed.). New York: Academic Press.

Davis, M. H., Hall, J. A., \& Meyer, M. (2009). The first year: Influences on the satisfaction, involvement, and persistence of new community volunteers. Personality and Social Psychology Bulletin, 29, 248-260.

Farh, J., Tsui, A. S., Xiu, K., \& Cheng, B. (2008). The influence of relational demography and guanxi: The Chinese case. Organizational Science, 9, 471-488.

Farrel, J. M., Johnston, M. E., \& Twynam, G. D. (1998). Volunteer motivation, satisfaction, and management at an elite sporting competition. Journal of Sport Management, 12, 288-300.

Finkelstein, M. A. (2008). Volunteer satisfaction and volunteer action: A functional approach. Social Behavior and Personality, 36, 9-18.

Finkelstein, M. A., Penner, L. A., \& Brannick, M. T. (2005). Motive, role identity, and prosocial personality as predictors of volunteer activity. Social Behavior and Personality, 33, 403-417.

$\mathrm{Fu}, \mathrm{Y} .$, \& Cai, X. (2009). A selected collection of the doctrine of the mean. Hong Kong: Xianggang Han Lin Publishing.

Galindo-Kuhn, R., \& Guzley, R. M. (2001). The volunteer satisfaction index: Construct definition, measurement, development, and validation. Journal of Social Service Research, 28, 45-68.

Green, S. K., Aarons, A., \& Cross, R. (1984). Volunteer motivation and its relationship to satisfaction and future volunteering. Paper presented at the annual meeting of the American Psychological Association, Toronto, Ontario.

Grimm, R., Dietz, N., Foster-Bey, J., Reingold, D., \& Nesbit, R. (2006). Volunteer growth in America: A review of trends since 1974. Washington, DC: Corporation for National and Community Service.

Haher, T. R., Gorup, J. M., Shin, T. M., Homel, P., Merola, A. A., Grogan, D. P., et al. (1999). Results of the Scoliosis research society instrument for evaluation of surgical outcome in adolescent Idiopathic Scoliosis. Spine, 24, 1435-1440.

Hidalgo, M. C., \& Moreno, P. (2009). Organizational socialization of volunteers: The effect on their intention to remain. Journal of Community Psychology, 37, 594-601.

Hong, S., Morrow-Howell, N., Tang, F., \& Hinterlong, J. (2009). Engaging older adults in volunteering. Nonprofit and Voluntary Sector Quarterly, 38, 200-219.

Houle, B. J., Sagarin, B. J., \& Kaplan, M. F. (2005). A functional approach to volunteerism: Do volunteer motives predict task preference? Basic and Applied Social Psychology, 27, 337-344. 
Hume, C., \& Hume, M. (2007). The strategic role of knowledge management in nonprofit organizations. International Journal of Nonprofit and Voluntary Sector Marketing, 13, 129-140.

Independent Sector. (2001). Giving and volunteering in the United States: Key findings. Washington, DC: Independent Sector.

Legislative Council Secretariat. (2009). Fact sheet: Fostering growth in youths via volunteerism. Hong Kong: Legislative Council Secretariat. (In Chinese).

Millette, V., \& Gagne, M. (2008). Designing volunteers' tasks to maximize motivation, satisfaction and performance: The impact of job characteristics on volunteer engagement. Motivation and Emotion, 32, 11-22.

Morgan, P. J., Cleave-Hogg, D., DeSousa, S., \& Tarshis, J. (2004). High-fidelity patient simulation: Validation of performance checklists. British Journal of Anaesthesia, 92, 388-392.

Moss, S., Prosser, H., Costello, H., Simpson, N., Patel, P., Rowe, S., et al. (1998). Reliability and validity of the PAS-ADD checklist for detecting psychiatric disorders in adults with intellectual disability. Journal of Intellect Disability Research, 42, 173-183.

Netting, F. E., Nelson, H. W., Borders, K., \& Huber, R. (2004). Volunteer and paid staff relationships: Implications for social work administration. Administration in Social Work, 28, 69-89.

Nunnally, J. C. (1978). Psychometric theory (2nd ed.). New York: McGraw-Hill.

Omoto, A. M., \& Snyder, M. (1995). Sustained helping without obligation: Motivation, longevity of service, and perceived attitude change among AIDS volunteers. Journal of Personality and Social Psychology, $68,671-686$.

Penner, L. A., \& Finkelstein, M. A. (1998). Dispositional and structural determinants of volunteerism. Journal of Personality and Social Psychology, 74, 525-537.

Preston, J. B., \& Brown, W. A. (2004). Commitment and performance of nonprofit board members. Nonprofit Management \& Leadership, 15, 221-238.

Reader, T. W., Flin, R., Mearns, K., \& Cuthberston, B. H. (2007). Interdisciplinary communication in the intensive care unit. British Journal of Anaesthesia, 98, 347-352.

Sturmey, P., Newton, J. T., Cowley, A., Bouras, N., \& Holt, G. (2005). The PAS-ADD checklist: Independent replication of its psychometric properties in community sample. British Journal of Psychiatry, 186, 319-323.

Taylor, T. P., \& Pancer, S. M. (2007). Community service experiences and commitment to volunteering. Journal of Applied Social Psychology, 37, 320-345.

Thurstone, L. L. (1953). Thurstone temperament schedule. Chicago: Science Research Associates.

Tschirhart, M., Mesch, D. J., Perry, J. L., Miller, T. K., \& Lee, G. (2001). Stipended volunteers: Their goals, experiences, satisfaction, and likelihood of future service. Nonprofit and Voluntary Sector Quarterly, $30,422-443$.

World Values Study Group. (1990). World Values Survey, 1990 (computer file) ICPSR version. Ann Arbor, MI: Institute for Social Research (producer).

Wu, J., Lo, T. W., \& Liu, E. S. C. (2009). Psychometric properties of the volunteer functions inventory with Chinese students. Journal of Community Psychology, 37, 769-780. 